\title{
APPROCHES DU POLITIQUE
}

L'État moderne : genèse. Bilans et perspectives. Dir. Jean-Philippe GenET. Actes du colloque tenu au C.N.R.S., Paris, 19-20 sept. 1989. Paris, Éd. du Centre national de la recherche scientifique, $1990.16 \times 24,352 \mathrm{p}$.

La parution de cet ouvrage est une étape d'une recherche qui se termine dans un cadre français pour se poursuivre désormais à l'échelle européenne. Le soustitre - Bilans et perspectives - prend toute sa valeur car il ne saurait y avoir solution de continuité entre l'entreprise nationale et l'entreprise européenne puisque leurs horizons restent les mêmes. Ce sont eux justement qui constituent la section I de l'ouvrage composée des contributions d'Adeline Rucquoi, "Genèse médiévale de l'Espagne moderne : du pouvoir à la nation (1250-1516)", de Christian Hermann, "Multinationale Habsbourg et universalisme chrétien ", de Jean Bérenger et Daniel Tollet, « La genèse de l'État moderne en Europe orientale, synthèse et bilan ", de Michel Balard, "État et colonisation au Moyen Âge. Bilan et perspective ", enfin d'Henri Bresc et Christiane Veauvy, "Genèse de l'État moderne en Méditerranée ». Tous ces articles présentent une réflexion méthodologique et critique sur des États aux structures différentes dont les évolutions spécifiques tranchent avec le modèle établi. Originalité providentielle pour le chercheur puisqu'elle l'oblige à affiner ses enquêtes dans l'espace et le temps et à repérer dans une évolution ces fameux « tournants " où les événements jouent un rôle de premier plan. Dans cette perspective, on peut regretter que les deux contributions de Michel Balard et d'Henri Bresc et Christiane Veauvy n'aient pas été placées en tête des "Horizons".

Consacrée aux « Moyens de l'État», la seconde section de l'ouvrage contient les articles de Mireille Corbier, " De la razzia au butin. Du tribut à l'impôt. Aux origines de la fiscalité : prélèvements tributaires et naissance de l'Etat », de Georges Dépeyrot, " Production monétaire et État : bilan et perspectives », et de Jean-Claude Hocquet, "L'impôt du sel contre la modernité de l'État". Ces études abordent une question classique mais encore mal connue tant sa théorie et sa pratique peuvent être multiformes, surtout quand elles sont saisies dans une longue durée jalonnée de termes qui scandent une évolution : razzia, butin, tribut, impôt... en attendant les contributions de l'époque contemporaine. À cet égard, il convient de méditer le rapport de synthèse de Mireille Corbier, riche de la

Revue de synthèse : IV S. Nos 3-4, juil.-déc. 1991. 
réflexion comparative issue de séances de séminaires consacrés à la fiscalité. La même démarche sous-tend les différents thèmes abordés par Jean-Claude Hocquet qui fait des usages fiscaux du sel des révélateurs du fonctionnement de l'État selon les âges et selon les pays avec pour risques permanents les réactions hostiles des populations.

La troisième section "Agents et adversaires " qui prend en compte les hommes étudiés dans leur diversité de fonctions, d'intérêts et de refus, comprend les contributions de Hélène Millet, «Les chanoines au service de l'État : bilan d'une étude comparative ", de Robert Descimon, "Modernité et archaïsme de l'État monarchique : le Parlement de Paris saisi par la vénalité ( $\mathrm{xv}^{\mathrm{e}}$ siècle) », de Jean Nagle, "L'officier " moyen" dans l'espace français de 1568 à 1665 ", de JeanMarie Constant, «Les partis nobiliaires et le développement de l'État moderne : le rôle de la noblesse seconde", et de Jean Nicolas, "Pouvoir et contestation populaire à l'époque du second absolutisme (bilan provisoire d'une enquête)" ". Nous sommes ici au cour de la problématique et les auteurs, conscients de cette responsabilité, insistent sur le parcours méthodologique de leurs recherches. La complémentarité de leurs démarches et celle des acteurs qu'ils étudient durant des périodes proches ou identiques, permet au lecteur d'avoir une vue d'ensemble, sans doute provisoire, puisque ces enquêtes ne sont pas toutes achevées.

La quatrième section "Idéologie et représentation " clôt logiquement l'ouvrage puisqu'elle s'interroge sur les thèmes du pouvoir. Elle comprend les articles de Colette Beaune et André Vauchez, « Recherches sur le prophétisme en Occident ( $\mathrm{XII}^{\mathrm{e}}-\mathrm{XvI}^{\mathrm{e}}$ siècle) », de Marie-Henriette Jullien de Pommerol, "Textes politiques dans la bibliothèque des Papes d'Avignon ", d'André Gouron, "Continuité et discontinuité dans l'histoire du législatif médiéval : réflexions sur une recherche collective », de Michel Pastoureau, "Images du pouvoir et pouvoir des princes ", de Françoise Boudon, Monique Chatenet, Anne-Marie Lecoq, " La mise en scène de la personne royale en France au $x^{e}{ }^{e}$ siècle : premières conclusions ", et de Gérard Sabatier, "Rappresentare il principe, figurer l'État. Les programmes iconographiques d'État en France et en Italie du $x^{e}$ au xvir siècle ". La diversité de ces approches a de quoi dérouter le lecteur. Mais ce trouble est la meilleure introduction qui soit aux mises en scène des pouvoirs et du Pouvoir que proposent ces recherches porteuses à leur tour de nouvelles enquêtes. L'histoire de l'art se taille ici, avec bonheur, la part du lion puisque sans elle le pouvoir se prive de représentation et donc d'existence extérieure pour tous ceux qui ne le voient que de loin.

La conclusion de Jean-Philippe Genet, "L'État moderne : un modèle opératoire ? ", fait parfaitement le point de ces enquêtes " en marche ", prometteuses de nouvelles recherches pour les historiens. Ils ont déjà à leur disposition dans cet ouvrage les annexes avec sources et bibliographies des travaux des différentes équipes. Leur consultation est précieuse. C'est donc, à tous égards, un ouvrage de référence que nous offrent les chercheurs qui se sont penchés et se penchent encore sur la genèse de l'État moderne. 
Anne Lombard-Jourdan, "Montjoie et saint Denis!" Le centre de la Gaule aux origines de Paris et de Saint-Denis. Paris, Presses du Centre national de la recherche scientifique, 1989. $16 \times 22,3,392$ p., bibliogr., ill.

Les bons livres d'histoire sont trop peu nombreux à paraitre pour qu'on puisse se permettre de bouder son plaisir à la lecture de celui de Madame LombardJourdan. L'auteur fait la démonstration qu'en usant d'une langue simple et claire elle sait rendre accessibles au profane les déductions les plus subtiles que lui inspire sa haute érudition, sans jargon prétentieux ni développements boursouflés. Au terme d'années de quête et de réflexions (ses premiers articles préparatoires parurent au début des années 1970), la vraie science s'écrit de façon limpide. Cette belle leçon se trouve heureusement confortée par la qualité matérielle du livre, vrai produit fini de la meilleure imprimerie moderne qui sait concilier netteté dans la mise en page et clarté dans la typographie. Dix-sept cartes et croquis, quarante-trois illustrations photographiques en noir et blanc, jamais gratuites, étayent à chaque pas, quand cela s'avère nécessaire, le développement de la pensée de l'auteur, bien insérés au regard des paragraphes qui les appellent comme autant de preuves iconographiques ou cartographiques. Que les Presses du C.N.R.S. soient remerciées du soin mis dans cette édition, dont on regrettera seulement le prix de vente sans doute dissuasif pour un lecteur non averti, encore ceci est-il la rançon de la qualité éditoriale.

L'argument du livre est simple : à l'époque gauloise le centre religieux et mythique de la Gaule était localisé en Ile-de-France, plus précisément dans la vaste plaine du Lendit. L'indépendance brisée du pays ne fit pas se perdre le souvenir de ce lieu sacré qu'honorèrent tour à tour Jules César, des empereurs romains puis les conquérants barbares, et que finalement « le premier apôtre des Gaules " recouvrit de son nom pour y avoir encouru le martyre. Désormais saint Denis s'affirmera comme l'un des protecteurs tutélaires de la monarchie française, tandis que l'ancien enclos druidique gardera sa fonction originelle de Montjoie, de " protège-pays " (du francique*mund-gawi ?) : l'abbaye de Saint-Denis, dont l'importance cruciale dans le développement tant politique qu'idéologique de la monarchie capétienne ne sera contestée par personne, serait ainsi l'héritière du "plus beau temple du monde " que Constantin visitait encore en 360.

Extraordinaire permamence des lieux sinon des corps de croyance constituée! Si l'on accepte l'idée directrice de l'auteur, c'est tout un pan de l'histoire de France qui vient à s'éclairer, projetant des lueurs neuves sur notre présent. Les géographes ont depuis longtemps mis en évidence les effets du centralisme parisien et les dysfonctionnements qu'il génère aujourd'hui, mais ils hésitent toujours quant aux raisons profondes qui assurèrent le triomphe millénaire de Paris. La réponse à ces interrogations pourrait bien résider dans le livre de Madame Lombard-Jourdan : malgré les vicissitudes de l'histoire des peuples et des pouvoirs successifs, depuis deux mille ans le centre de gravité du pays se situerait dans la plaine de France, dans l'actuelle commune de Saint-Denis, sur l'emplacement de la tombe de l'ancêtre héroïsé devenu le protecteur attitré du pays. Au cœur de la cité des Parisii, la Montjoie comme son équivalent de Lutèce/Paris auraient bénéficié d'un statut spécifique à travers les âges, statut que les ducs des Francs rober- 
tiens puis les rois de France capétiens devaient mettre au service de leurs ambitions, érigeant le second site en capitale politique et le premier en forteresse dynastique dûment organisée autour de la nécropole royale (ouverte dès les premiers Mérovingiens qui plus est) et de ses ateliers d'écriture chargés de l'exaltation de la geste royale et nationale.

Cette continuité imposée à tous les dirigeants de la Gaule (un temps seulement éclipsée par Lyon), puis exaltée par ceux de la France jusque dans le jacobinisme contemporain pose un problème de fond, celui de la nature même du substrat historique français : la part de l'héritage gaulois n'y est-elle pas couramment minorée ? Beaucoup d'arguments tendraient à le rappeler, nous sommes bien les petitsenfants de la Gaule, même si l'arrière-fond indigène fut trop souvent rejeté dans les ténèbres de l'oubli. Dans la perspective d'une réhabilitation d'un passé obscur et prégnant à la fois, commodément qualifié de celtique (car il pourrait tout autant surgir des temps préhistoriques), la savante étude de Madame Lombard-Jourdan apporte une contribution de poids qui forcera même les plus sceptiques à y réfléchir. Son travail d'historien me paraît relever d'une double démarche bien maîtrisée, la chartiste tant chaque indice est passé au crible d'une critique rigoureuse dont l'auteur apprit les règles exigeantes dans l'illustre école, et la policière tant les éléments du puzzle historique s'ordonnent de façon serrée au fil d'un discours heuristique. L'ensemble se lit donc comme une enquête policière sur un passé enfoui, dont l'action rebondit d'indice en indice, d'âge en âge, pour déboucher sur le plateau assuré des relations privilégiées entretenues par l'abbaye de Suger avec la monarchie française. Lecture passionnante, parfois haletante lorsque s'enchaînent les rapprochements érudits entre toutes les pièces d'un palimpseste que l'auteur prend plaisir à nous restituer aux dépens des bons moines qui, eux, prirent grand soin jadis d'en brouiller les pistes et d'effacer les indices trop crus d'un passé païen inacceptable à leurs yeux...

On l'aura compris : par sa reconstruction érudite qui s'épanouit en une quête des origines perdues de la France, Montjoie et saint Denis tranche sur la savante tristesse de trop de productions universitaires. Certes les micro-spécialistes pourront discuter certaines affirmations de détail, d'autres étaler leur scepticisme foncier sur des siècles encore bien obscurs ou discuter la philosophie générale du livre. Il reste que jamais cette étude ne laisse son lecteur indifférent. Puisse-t-elle contribuer ainsi à provoquer des débats de fond, vrais gages de renouvellements et de progrès vers une appréhension plus exacte du passé! C'est le propre des grands livres que d'y réussir à l'échelle d'une génération.

Jean-Christophe CASSARD.

Violence et contestation au Moyen Age. Actes du $114^{\mathrm{e}}$ Congrès national des sociétés savantes, Section d'histoire médiévale et de philologie, Paris, 1989. Paris, Comité des travaux historiques et scientifiques, $1990.16 \times 24,358 \mathrm{p}$.

Les organisateurs du $114^{e}$ Congrès national des sociétés savantes (Paris, 1989) avaient proposé aux contributeurs d'organiser leur réflexion en fonction de deux 
thèmes novateurs, la violence et la contestation au Moyen Âge, qui nous sont présentés dans l'introduction de François-Olivier Touati (que suit une précieuse bibliographie). Le résultat n'est pas à la hauteur des espérances par suite du grand libéralisme du comité qui accepte des communications sans rapport avec le thème de l'année : la tâche du recenseur n'en est pas facilitée ! Et il n'est pas sûr que la recherche historique y gagne vraiment. Nous regrouperons nos impressions de lecture sous trois rubriques : violences rurales, violences urbaines et, d'abord, situations dont la violence est absente.

1. John Ottaway reprend le dossier de la charte de fondation de l'abbaye de la Trinité de Beaulieu-lès-Loches, connue au travers de deux actes conservés par des copies modernes. L'examen des témoins cités lui permet de les replacer dans un contexte du milieu du xI ${ }^{e}$ siècle, sous le règne de Geoffroy Martel et non de son père Foulques Nerra fondateur de l'abbaye en 1004. En fait, ces actes sont certainement composites : l'examen minutieux de leurs clauses permet à l'auteur de distinguer plusieurs strates de rédaction depuis le début du xi ${ }^{e}$ siècle, le milieu de ce siècle quand Geoffroy entend faire de la Trinité la nécropole de son père décédé en Terre Sainte en 1039, et l'année 1163 qui vit probablement la mise en forme définitive du texte. Et ces strates chronologiques correspondent effectivement aux différentes campagnes architecturales sur l'abbaye et permettent de mieux cerner les libertés concédées aux bourgeois de Loches dans le courant du $\mathrm{XI}^{\mathrm{e}}$ siècle, au terme d'une étude diplomatique savante et nuancée.

Au Sud de l'Aisne, entre l'Oise et Soissons, l'importante seigneurie de Pierrefonds regroupait une quarantaine de villages ou hameaux. Entrée dans le domaine royal sous Philippe-Auguste, elle fut concédée en douaire à la reine Blanche en 1240 : celle-ci décida d'affranchir ses serfs en 1252, initiative bientôt confirmée par son fils Louis IX (en septembre 1255). Louis Carolus-Barre publie le dossier documentaire de cette manumission (chartes originales, listes des serfs libérés établies village par village), ce qui lui donne l'occasion de présenter les bénéficiaires, d'étudier leurs anthroponymes parfois pittoresques, projetant ainsi sur la paysannerie d'Ile-de-France au XII' siècle d'intéressants éclairages. 890 serfs sont nommément cités, la fortune de chacun étant estimée (puisqu'une taxe d'un vingtième devait être levée) : cette société rurale apparaît déjà bien différenciée et n'a besoin de nul recours à la violence pour obtenir la liberté personnelle dans un contexte de paix et de relative prospérité matérielle.

Nulle violence non plus dans l'attitude des États du Dauphiné devant l'impôt, telle que retracée par Isabelle Vernus-Moutin. Jusqu'en 1439, l'assemblée delphinale parvient sans trop de heurts à s'organiser face à des dauphins absentéistes qui se déchargent sur elle de la levée des impôts mais contrôlent strictement leur utilisation. Au contraire, avec Louis II (le futur Louis XI), le dauphin se fait très présent et autoritaire : si les États ne bronchent pas devant ce changement d'attitude, c'est que le prince sait fermer les yeux sur certains abus fiscaux et distribuer aux ténors de l'assemblée de généreuses pensions. Choyés par le pouvoir, les grands préfèrent abdiquer toute velléité politique dans une assemblée devenue simple chambre d'enregistrement.

2. Dans son Autobiographie rédigée vers 1114-1115, la figure de Thomas de Marle apparaît à Guibert de Nogent comme emblématique de la violence seigneuriale la plus débridée, la plus ordinaire. Et le talent de générations d'historiens lui 
a maintenu cette peu enviable réputation. Mais l'est-elle en vérité ? FrançoisOlivier Touati fait remarquer que Guibert accumule dans un présent épais d'une trentaine d'années d'observations tous les types de violences dont il a eu écho, confondant sciemment faits divers crapuleux et attentats politiques. Regardés de près, les excès de Thomas de Marle peuvent s'expliquer par les seuls impératifs de ses entreprises militaires : chez lui, il ne se conduit pas en seigneur violent et, d'ailleurs, Guibert n'évoque jamais la lourdeur de sa justice domaniale en tant que telle. En fait, les racines de son discours apocalyptique sur les cruautés sans borne d'un monstre seraient à rechercher, plus que dans une violence assez impalpable, dans la personnalité à la sensibilité d'écorché vif de l'auteur : fils mal aimé, voué très jeune à l'état ecclésiastique, il adhéra avec enthousiasme à l'idéal de la réforme grégorienne. Son espérance déçue d'un monde conçu sur le plan divin le pousse inexorablement à peindre sous des couleurs extrêmes la société qui l'entoure, où le mal lui paraît triompher partout : par ses artifices son écriture le conforte dans cette appréhension pessimiste du monde qui justifie un appel pressant à la pénitence. Cet article fournit une intéressante approche de psychologie historique, fondée sur une méthodologie prudente, mais peut-on la généraliser et appréhender une facette de l'homme commun de ces temps au travers d'une personnalité aussi exceptionnelle que Guibert de Nogent?

Les tuchins ne sont-ils qu'une variante régionale des Jacques? Pierre Charbonnier relit le maigre dossier documentaire les concernant, surtout des lettres de rémission : elles laissent entrevoir un mouvement assez complexe, auvergnat et languedocien, actif entre 1360 et 1390 en gros, mobilisant essentiellement des paysans (comme les Jacques) mais aussi des citadins, voire quelques bourgeois. Des motivations sociales ne peuvent rendre compte seules du tuchinat. Refusant le simplisme des rares études antérieures, l'auteur remarque que les documents du temps sont eux-mêmes ambigus, le fait d'avoir tué un tuchin pouvant amener à rechercher la grâce du roi : il ne s'agit donc pas de simples bandits ruraux, mais plutôt de résistants à la présence anglaise en Auvergne, à celle des hommes d'armes en général en Languedoc. Résistants, assez bien organisés en troupes capables d'actions militaires ponctuelles et réfléchies, les tuchins n'acceptent pas les traités ou " patis » signés entre des autorités locales débordées et l'occupant : ils attaquent les Anglais en toute bonne conscience puisque le roi condamne le principe de ces accords locaux. Certes des tuchins verseront dans le banditisme et leur action se trouvera vidée de son contenu patriotique par la conclusion des trèves officielles : il n'en demeure pas moins qu'ils préfigurent les paysans normands insurgés après l'occupation de leur province en 1418 et que les Anglais traitaient de « brigands ». Bandits ou patriotes ? Terroristes ou héros? Les termes de cette alternative médiévale demeurent, hélas, très modernes...

Les révoltes paysannes en Basse-Bretagne à la fin du $x^{e}$ siècle sont abordées par deux études : celle de Claude Fagnen n'apporte ni document nouveau ni interprétation nouvelle, à l'inverse de celle de Michel Nassiet qui explique bien les mécanismes de la tenure congéable et qui, surtout, par une analyse serrée des rares indications disponibles, dégage une chronologie renouvelée du soulèvement comouaillais qui se serait déroulé en deux temps. La responsabilité des vicomtes de Rohan dans ces tragiques événements est bien soulignée, comme l'est la violence de la répression seigneuriale. 
Les « rustauds" de la guerre des paysans d'Alsace échappent au Moyen Âge stricto sensu tant pour leurs motivations religieuses réformées que pour leur art militaire spécifique : il n'existe, en effet, aucune armée paysanne médiévale comparable, capable de s'entraîner, de se mobiliser et de se battre sous ses propres chefs. La situation particulière de l'Alsace dans la géographie politique de l'époque, l'organisation ancienne de ses efficaces milices villageoises, son appartenance à un monde germanique grand fournisseur de mercenaires, les lansquenets, tous ces facteurs réunis font qu'en 1525 les paysans insurgés se rassemblent sans hésitation en armées véritables, capables de sens tactique et assez bien équipées en armes à feu, handicapées toutefois par l'absence d'une cavalerie. Malgré son incontestable savoir-faire, l'infanterie paysanne sortira battue des batailles rangées que lui imposeront les soldats professionnels appelés à la rescousse par le duc de Lorraine : Georges Bischoff retrace avec érudition la courte épopée de ces légions rurales et s'interroge sur l'organisation de la société qui a permis l'éclosion de pareille entreprise.

3. Abordant la sphère des révoltes urbaines, Élisabeth Lalou procure une utile recension des multiples soulèvements qui ponctuèrent de façon souvent dramatique la vie citadine depuis la fin du XII' siècle jusqu'aux premières décennies du siècle suivant. L'auteur, s'appuyant exclusivement sur la littérature historique disponible, ordonne son propos en fonction d'une classification claire et simple, distinguant les révoltes contre le seigneur traditionnel, celles contre le roi et sa fiscalité, contre l'Inquisition à la suite des prêches de Bernard Delicieux en Toulousain et, enfin, ce seront les plus acharnées et les plus longues à réduire, les violences contre les oligarchies bourgeoises contestées dans leur gestion et leur pouvoir municipaux par les couches nouvelles du popolo minuto, en Flandre et en Artois (Saint-Omer) surtout. Cet impressionnant catalogue (et sans doute les archives n'ont-elles pas livré tous leurs secrets) complète les recherches antérieures entreprises sur les deux derniers siècles du Moyen Âge et permet de nuancer la nouveauté du phénomène insurrectionnel urbain qui n'éclot pas d'un coup à l'entrée des décennies difficiles du $\mathrm{xIV}^{\varepsilon}$ siècle; la matière réunie par l'auteur se trouve éclairée par ses quelques remarques finales qui mériteraient un approfondissement.

C'est à quoi s'attelle Bruno Galland à partir d'un exemple rhodanien : trois villes comparables par leur situation commerciale avantageuse, leurs fonctions portuaires importantes, leur statut de sièges archiépiscopaux, soit Vienne, Lyon et Romans, voient leurs histoires analysées et comparées depuis la fin du xiI ${ }^{e}$ jusqu'au début du xIv siècle. Malgré des différences sensibles dans les rapports de force locaux, l'auteur peut mettre en évidence deux vagues revendicatives bien distinctes, séparées par une cinquantaine d'années calmes : la première voit l'affirmation des exigences économiques face au seigneur traditionnel, l'archevêque ou le chapitre à Romans; la seconde est plus politique, le corps de ville tentant d'accroître des libertés anciennes. Cette étude régionale apporte une contribution intéressante à l'établissement d'une typologie raisonnée des mouvements urbains dans toute leur complexité et leurs motivations changeantes selon les époques.

Sur le thème voisin de la violence dans la ville, Robert-Henri Bautier publie et analyse le dossier policier d'une rixe survenue à Orléans en 1284 et opposant des étudiants clercs au prévôt du roi. Vingt-trois témoignages dûment enregistrés 
éclairent ces événements graves : le sang des officiers royaux n'a pas seulement été répandu, la personne même du roi a été injuriée au prétexte de la Croisade d'Aragon que Philippe le Bel venait juste de décider. Les injures ont été redoublées de gestes obscènes sans ambiguiité quant au respect porté à la monarchie. Les armes employées et les préparatifs de siège ne laissent, enfin, aucun doute quant au caractère prémédité de ces incidents sans rapport avec un banal chahut d'étudiants. La répression sera terrible après la prise d'assaut de l'hôtel particulier dans lequel les neuf trublions appréhendés s'étaient retranchés. Par ses connotations antimonarchiques, cette affaire rejoint la problématique de l'article de Pierre Charbonnier dans le présent volume et, plus généralement, les travaux de Colette Beaune sur « la naissance de la nation France » : la supposée allégeance des Français à la couronne était-elle aussi profonde qu'on a coutume de le dire ? Leur patriotisme était-il si assuré ? Peut-être, suggère l'auteur, la cristallisation du sentiment national ne pouvait-elle intervenir qu'à la faveur d'une crise exceptionnellement grave et durable : la guerre de Cent ans, avec tous les déchirements nés du conflit franco-anglais. Auparavant, les choses apparaissent moins tranchées et le roi lui-même peut être moqué et critiqué sans égards particuliers, même dans l'une de ses bonnes villes au cœur de ses domaines patrimoniaux.

Jean-Christophe CASSARD.

Peter Gwy,, The King's Cardinal: The Rise and Fall of Cardinal Wolsey. Londres, Barrie and Jenkins, 1990. $16 \times 24$, xxii-666 p.

Que sait-on de Thomas Wolsey? De l'homme privé en définitive très peu : sa date de naissance est incertaine, son physique discuté, ses goûts et sa personnalité nous échappent. De l'homme public, à l'évidence, bien plus mais, nous assure Peter Gwyn, le cardinal politicien que nous croyions connaitre n'est pas le véritable Wolsey. D'une part, parce qu'il a été la cible de l'hostile propagande des protestants et des Whigs (de Polydore Vergil à Sir Geoffrey Elton) dont ni George Cavendish, ni Mandell Creighton, ni plus récemment et dans une moindre mesure Jack Scarisbrick n'ont réussi à annihiler les durables effets. D'autre part, parce que les historiens, influencés par les sources disponibles, ont accordé trop d'importance à la politique extérieure de Wolsey au détriment de sa politique intérieure.

The King's Cardinal est donc une tentative de réhabilitation " révisionniste", en six cent quarante-sept denses pages, de l'homme orchestre des vingt premières années du règne d'Henry VII. Le résultat emporte-t-il l'adhésion? Je n'en suis pas entièrement convaincu.

L'architecture même du livre est discutable : ni chronologique, ni thématique, ni logique, elle conduit à de nombreuses répétitions, à des retours en arrière déroutants et finit par lasser le lecteur le mieux disposé. Ajoutons que le style confus et hésitant de l'auteur n'engage guère à l'effort de lecture. De surcroît, écrire que l'on convie le lecteur à suivre " une sorte d'histoire policière » (p. xxii) est peut-être justifiable, mais n'est pas Agatha Christie qui veut et trop de pistes 
mènent nulle part (ainsi celle du complot contre Wolsey en février-avril 1518, p. 89-92).

Mais l'essentiel est ailleurs. Incontestablement Peter Gwyn a raison de souligner avec insistance que Wolsey était une créature d'Henry VIII et qu'il s'évertua à servir et à défendre les intérêts du roi avant les siens propres. Il ne pouvait en être autrement. Fils de boucher, sans assise sociale, Wolsey n'aurait jamais pu atteindre, et rester, au sommet de l'État sans bénéficier de la protection et de la faveur royales et ce serait profondément méconnaître la structure du pouvoir dans l'Angleterre d'Henry VIII que d'affirmer le contraire. La chute de Wolsey en est la preuve éclatante. De même, il est tout à fait exact que Wolsey était un travailleur infatigable, un habile politicien et un négociateur hors pair. Les ambassadeurs français en firent l'amère expérience en août 1525 lors de la signature des traités du More. À la dernière heure, Wolsey obtint de Brinon et Passano l'avantageux accord financier qu'il souhaitait et que Louise de Savoie voulait lui refuser. Les Français avaient cédé.

Pourtant, à trop vouloir dresser un portrait flatteur de son héros, Gwyn distord parfois la réalité des faits. Wolsey joua un rôle capital sur la scène internationale et il est tout à fait exact qu'il fut l'architecte principal de la « paix universelle » de 1518 et de la " paix perpétuelle " de 1527 entre la France et l'Angleterre. Pourtant, je crois exagéré d'écrire que cela traduit le « rôle dominant de l'Angleterre dans cette relation [avec la France] " (p. 94) ou que l'Angleterre jouait " un rôle dominant dans les affaires européennes " (p. 101). L'accession de François $\mathrm{I}^{\text {er }}$ à la couronne de France, celle de Charles Quint à l'Empire, Marignan, Pavie et le Sac de Rome eurent tout de même des conséquences considérables et durables sur la scène européenne dont le royaume Tudor, étranger à ces événements, dut bien s'accommoder. En dépit des allégations de Gwyn, l'Angleterre de Wolsey ne dictait pas sa loi à François $I^{\text {er }}$ et Charles Quint. Sinon, pourquoi l'échec du projet d'invasion de la France sitôt le désastre de Pavie connu ou pourquoi l'échec du divorce? Peter Gwyn réfute également la thèse, défendue par Scarisbrick, selon laquelle Wolsey voulait la paix et Henry VIII la guerre, en montrant que le propos central de la politique de Wolsey était l'accroissement de la gloire du roi. La paix et la guerre n'étaient que des instruments au service de cette gloire : croire à l'une ou à l'autre n'était pas la préoccupation du cardinal. L'argumentation de Gwyn n'est pas sans fondements mais elle me semble sous-estimer l'attachement de Wolsey aux idéaux humanistes et celui d'Henry VIII à revivre les chevauchées victorieuses d'Henry V. Elle devient excessive lorsque l'auteur écrit que Wolsey « n'était pas très intéressé par l'équilibre des pouvoirs [en Europe]» (p. 84) et qu'en 1525, comme en 1516, les ardeurs guerrières de l'Angleterre n'étaient qu'un « rideau de fumée diplomatique destiné à tromper ses alliés (p. 84 et 398-401). Suprême habilité diplomatique de Wolsey ou erreur d'appréciation de Gwyn? Je penche pour la seconde hypothèse. D'une part, parce que la thèse de Gwyn revient à dire qu'il ne faut accorder que peu de crédibilité aux propos publics de Wolsey et d'Henry VIII. D'autre part, parce que leurs contemporains crurent à la paix en 1518 et en 1527 et à la guerre en 1513 et en 1522-1525. Ainsi, en refusant de payer l'« Amicable Grant » de 1525, la population s'opposa à une nouvelle invasion de la France. 
En ce qui concerne la politique intérieure de Wolsey, Peter Gwyn n'apporte guère d'éléments neufs. Ainsi, la controverse autour du programme de réformes de 1519 et du fait qu'il ne fut pas mis en œuvre, est en grande partie éludée. Par ailleurs, Peter Gwyn soutient à plusieurs reprises que Wolsey était « doué d'une grande habilité à s'entendre avec les gens [...] et qu'il s'efforça toujours de se concilier la noblesse anglaise " (p. 11). Il estime ainsi que la chute de Wolsey n'est pas due aux mancuvres d'une faction aristocratique (chap. 13), ni que sa rapide arrivée au pouvoir ne suscita pas d'oppositions ou de jalousies (en particulier, la rivalité Wolsey-Warham est écartée simplement sur la foi d'une correspondance courtoise entre les deux hommes après le départ de l'archevêque). Ce n'est pas très convaincant et c'est faire peu de cas des travaux de David Starkey et d'Eric Ives. Que la survie politique - et physique - de Wolsey dépendît en dernier ressort de la volonté royale ne signifie pas pour autant que le cardinal fût à l'abri des luttes politiques au sommet de l'État.

En dépit de ces critiques, The King's Cardinal est un livre intéressant. C'est la première biographie de Wolsey depuis celle d'Albert Pollard parue en 1929 et, non seulement elle incorpore les travaux récents sur la période, mais elle a aussi le grand mérite d'être fondée sur une relecture des documents originaux. Peter Gwyn a réussi à corriger certains excès de l'historiographie (aux normes de son époque, Wolsey n'était pas particulièrement corrompu) et à replacer Wolsey dans le débat sur le règne d'Henry VIII. Ce n'est pas négligeable.

Charles Giry-Deloison.

Denis Crouzet, Les Guerriers de Dieu, La violence au temps des troubles de religion (vers 1525-vers 1610). Préf. Pierre Chaunu, av.-pr. Denis Richet, 2 vol. Seyssel, Champ Vallon, 1990. $16 \times 24,793$ p.-pl. et 738 p.-pl., bibliogr. (« Époques ").

Les guerres de religion n'ont pas commencé le jour de Vassy, pour se clore à l'édit de Nantes : elles démarrent dès les années 40 , sinon avant, et se prolongent jusqu'en 1610. Les guerres de religion ne sont pas un conflit socio-politique à prétexte religieux, en dépit de l'opinion des " politiques " du xvi siècle et des positivistes des $\mathrm{XIX}^{e}$ et $\mathrm{Xx}^{e}$ siècles. Elles ne sont pas non plus le fruit pervers d'une aliénation de la religiosité, comme le pensent les historiens polémiques ou iréniques. Elles sont une "guerre de la fin du monde ", un temps où le temps et le temporel se dissolvent dans une geste sacrale. Ce surgissement brutal de pulsions irrationnelles ne peut pas relever d'une " histoire sans Dieu "!

Nous savions depuis Natalie $Z$. Davis que les violences de religion furent des actes rituels, spécifiques de chacune des confessions affrontées. Denis Crouzet va plus loin. Les deux violences sont les expressions et les moteurs de deux cultures qui se structurent au cœur des Français du $x^{\prime}{ }^{e}$ siècle. La Réforme entre Noyon, Nérac et Genève ne se vit pas comme à Wittenberg ou à Munster; elle est une entreprise pour sortir l'homme d'un monde enchanté et angoissant (le faire passer 
de l'univers de Bosch à celui, peut-être, de Vermeer). Très vite, et beaucoup plus tôt qu'on le dit, le catholicisme contre-attaque sur le plan spirituel, par un surenchantement panique : naissances monstrueuses, catastrophes naturelles, crimes affreux, progrès même de l'hérésie sont des signes, et disent l'ire de Dieu et l'imminence de la Parousie et du Jugement. La violence catholique se vit donc dans une ambiance eschatologique : elle investit des créatures faibles, pécheresses, mais repentantes, de la fonction d'instruments de Dieu. Elle révèle sous le masque trop humain des évangélistes des monstres difformes qu'il faut détruire à jamais, rendre à l'enfer. La violence réformée, elle, s'acharne sur les idoles, avec pour modèle le geste iconoclaste : elle réduit en poussière les dieux de pierre et de bois et les têtes tonsurées pleines d'illusions fumeuses. Elle ne se situe pas à la fin du monde, mais en son centre, contemporaine, par la conversion du pécheur élu, du moment indépassable où Dieu s'incarne en Homme. Le réformé est violent pour re-former la création; il sort du temps non vers l'eschatologie mais vers l'utopie.

Catholiques et protestants ne sont pas cependant délivrés de l'angoisse. Après l'état de grâce de toute violence sacrale, la durée reprend son cours, et le Mal est toujours là... C'est alors que la violence gagne le champ politique (mais sans changer de nature !). Malgré les directives et mises en garde de Genève, malgré l'entreprise d'occultation intellectuelle menée dès l'événement, les sources découvrent à l'historien perspicace une vague de fond subversive des hiérarchies sociales et étatiques, et très tôt le désir refoulé du régicide. Mais la révolution jouée et théâtralisée fait office de transfert pour une violence huguenote qui, en ce monde, ne peut que respecter l'ordre établi.

La Saint-Barthélemy (1572) est un paroxysme et un tournant. Comme on l'a toujours dit, mais pour d'autres raisons, et avec un autre partage des responsabilités. Ce paroxysme aux résultats incomplets déçoit les vainqueurs et désarçonne les vaincus. Une mélancolie inquiète s'empare des âmes, et on s'interroge sur la violence, la sienne, celle de l'ennemi, celle de Dieu... Le ministre Léry voit dans Sancerre assiégée et affamée des coreligionnaires devenir cannibales. Les catholiques " zélés " voient un peu partout des coreligionnaires « politiques » pactiser avec le Béarnais; ils voient au château de Blois le roi très chrétien assassiner le parangon du chevalier chrétien. La violence collective se sécularise après 1572 (mais les exemples donnés sont postérieurs à 1583 , voire 1589 ) : une soldatesque professionnalisée, souvent indifférente aux appartenances confessionnelles, s'en prend aux biens plus qu'aux personnes, à celles-ci plus qu'aux symboles. Politiques et huguenots "culpabilisent la violence » en tant que telle. Les ligueurs ciblent son usage (les traitres, le roi) et, surtout, l'intériorisent en développant une spiritualité autopénitentielle. La violence sacrale existe encore, mais tournée contre un ennemi lové dans l'âme. La « sainte union " ne se comprend bien que comme une union à Dieu et en Dieu. Et les circumambulations processionnelles disent ce repli sur l'intérieur, la cité juste, la paroisse, l'individu. L'indépassable durée du temps historique et l'inefficace du meurtre royal permettent la construction de nouveaux instruments idéologiques contre l'angoisse panique : le stoïcisme, l'absolutisme...

Les lignes qui précèdent ne prétendent pas rendre compte d'un livre qui défie tout résumé par ses dimensions comme par ses démarches. Le contenu de ses 
1500 pages bourgeonne, foisonne, et sème à tous vents ; même si une composition très concertée insère chaque développement dans une démonstration annoncée clairement en préalable et toujours suivie d'une conclusion où éclatent souvent de belles formules. Il y a un dessein global, une thèse défendue tout au long de l'ouvrage, et qu'explicite son sous-titre : la violence au temps des troubles de religion. Ce cap n'est jamais perdu de vue. Mais bien d'autres thèmes sont abordés, analysés, voire traités, qui, à eux seuls, pourraient faire autant de livres solides et neufs. Je me suis amusé à donner des titres à certains de ces livres dans le livre : "les guerres de religion revisitées", " au vrai coeur religieux du $x^{e}{ }^{e}$ siècle", "sous la Réforme la Révolution », " la contre-renaissance des dévots », " une archéologie de l'absolutisme", "vraie et fausse modernité du xvi" siècle", et même : " nouveaux essais sur le temps humain ", et "discours de la méthode historique $" . .$.

Un livre ambitieux, on le voit! Un livre qui brasse et embrasse beaucoup, et qui déstabilise, mais aussi conforte. Une somme d'érudition qui relit toutes les sources classiques, narratives et normatives, et en découvre d'autres : almanachs, canards, sermons d'un Artus Désiré... Une histoire qui réinterprète les événements canoniques (Saint-Bathélemy, mort de Henri III...) et qui en institue de nouveaux (l'iconoclasme de Guyenne en 1561 , les processions blanches de Champagne-Picardie en 1583-1584...). Un historien qui connaît, utilise, apprécie, mais aussi remet en question toute l'historiographie antérieure : un iconoclaste joyeux et ardent qui combine l'irrévérence la plus désinvolte avec la générosité la plus foncière, le tout bien tempéré par un constant humour qui s'exerce sur autrui comme sur soi-même. Un style, enfin, qui me rappelle curieusement celui de... Spinoza : une " concaténation " de propositions et d'arguments sans faille, ajustés de façon pesante, accablante, et puis, brusquement, le trou d'air frais d'une affirmation que rien n'étaye, sinon la sensation d'évidence ressentie subjectivement par celui qui la formule : "patet ", que D. Crouzet traduit par : "à mon avis », ou « je crois que $» . .$.

Qu'on permette au lecteur d'être lui aussi subjectif. Dans ce livre où rien ne laisse indifférent, il y a des thèmes qui m'ont été plus à cœur, et d'autres qui m'ont laissé plus réservé.

- J'ai apprécié ce livre comme une méditation documentée sur le problème fascinant d'une société qui se met à dysfonctionner, et où le plus solide s'écroule sous la pression du plus fragile... et celui de la reconstitution d'un ordre social qui n'a d'autre force au départ que l'adhésion des individus qui l'acceptent.

- J'ai aimé comment D. Crouzet traite par des analyses nuancées (plus que dans quelques formules tranchantes et pas toujours concordantes) la dialectique du spontané et de l'organisé dans la constitution de l'événement historique (vague iconoclaste, massacre de la Saint-Barthélemy, processions blanches, attentat de Jacques Clément...).

- Un parti pris m'a déçu, imposé sans doute par les nécessités de la démonstration : l'auteur a dressé face à face deux êtres de raison, l'homo catholicus et le reformatus, bardé chacun de sa vision du monde et de son style de violence. Mais dans les années 50 , ou même 80 , les deux communautés ne sont pas encore deux groupes culturels homogènes et hermétiques (même dans le Languedoc du XVII siècle, elles ne le seront pas totalement, comme l'a montré R. Sauzet). 
J'aimerais voir de plus près des êtres de chair et de sang, comme ce pamphlétaire huguenot qui interprète les signes de Dieu (I, 737); ou ce dom Lépaulart qui fraternise avec les hérétiques maîtres de sa bonne ville de Soissons... Ils sont les acteurs réels des guerres de religion. Les palinodies ne sont pas toujours (et jamais uniquement) fruits de l'opportunisme. Et les syncrétismes vécus plus que construits apportent quotidiennement angoisse et paix à des convertis qui n'ont pas tué vraiment «le vieil homme " et à des fidèles avides de désenchantement. Ces êtres qui eurent l'incohérence du vivant méritent la considération d'une histoire phénoménologique et post-positiviste.

En même temps que les Guerriers de Dieu, le hasard (mais existe-t-il ?) m'a fait lire La Grande discorde de Hichem Djait. La parenté de ces deux livres m'a frappé, par-delà la diversité de l'objet, du matériau, de l'approche. H. Djait, comme D. Crouzet, proclame que « la religion est un des monstres sacrés de l'histoire " et qu'une métahistoire est indissociable de l'histoire d'une société ou d'un pouvoir qui fait référence au sacré. Concordance qui, en cette fin de siècle, est un signe des temps. Mais aussi qui place les deux auteurs dans le sillage de la réflexion d'Alphonse Dupront. lls ne furent pas, au sens institutionnel, élèves de cet historien savant et subtil. Il n'en est que plus remarquable qu'ils se soient approprié la démarche de ce maître difficile et exigeant. D'autres ont été les disciples d'Alphonse Dupront. Denis Crouzet est peut-être - je pèse mes mots son successeur.

François Billacois.

Lancelot du Voisin de LA Popelinière, L'Histoire des histoires avec L'Idée de l'histoire accomplie: 1599. 2 vol. Paris, Fayard, 1989. $14 \times 22,3$, 405 p. et 361 p. ("Corpus des æuvres de philosophie en langue française ").

En publiant dans la collection " Corpus des œuvres de philosophie en langue française " L'Histoire des histoires de La Popelinière, les èditions Fayard mettent à la disposition des historiens des sciences sociales un grand texte du $x^{e}{ }^{e}$ siècle, qui prend place dans la collection à côté de ceux de Bodin et de Le Roy. Selon l'usage de cette collection, le texte est livré brut, sans introduction ni notes ; il est reproduit d'après l'édition de 1559 , dont l'orthographe est légèrement modernisée. Le premier tome, qui donne son nom à l'ensemble, est présenté par l'éditeur " comme une revue critique des historiens et de leurs histoires depuis l'Antiquité jusqu'à la fin de la Renaissance ». Le second tome, L'Idée de l'histoire accomplie contient une réflexion plus théorique sur la nécessité d'élargir l'enquête historiographique, en ne la bornant pas à une chronique des événements et en interrogeant tous les documents susceptibles de délivrer des renseignements sur les lois, les coutumes, les institutions religieuses du passé, de façon à parvenir à « la représentation de tout ". La Popelinière avait vivement conscience d'innover en procédant ainsi et, dès ses premières lignes, oppose la recherche de la vérité sous la conduite de la raison à la vénération paresseuse de l'Antiquité. La portée de ses 
travaux a été bien étudiée dans les ouvrages de D. R. Kelley, Foundations of Modern Historical Scholarship, New York et Londres, 1970 ; G. Huppert, L'Idée de l'histoire parfaite, Paris, 1973, traduit de l'américain; C. G. Dubois, La Conception de l'histoire en France au xvI siècle (1560-1610), Paris, 1977.

François LAPLANCHE.

Yves-Marie BerCE, Le Roi caché. Sauveurs et imposteurs, mythes politiques populaires dans l'Europe moderne. Paris, Fayard, 1990. 15,2 × 23,5, 483 p. bibliogr., index.

"Napoléon n'est pas mort à Sainte-Hélène " - une telle information sous la plume d'un historien aussi sérieux peut surprendre, mais l'auteur précise bientôt son propos : les peuples ont besoin de croire que les grands hommes ne meurent jamais. Chaque époque, chaque pays a suscité de telles déraisons. Le présent livre se veut une savante chronique de ces "espérances folles ». Nous sommes invités à un voyage dans l'imaginaire politique de l'Europe moderne.

Trois épisodes ont droit à un traitement circonstancié : trois chroniques de temps de trouble à l'extrême fin du $x^{\prime}{ }^{e}$ siècle. Le roi Sébastien de Portugal, mystérieusement disparu au cours d'un combat en 1578, et dont les partisans attendent le retour et espèrent une revanche sur l'occupant espagnol, est la première figure de " roi caché » présentée ici. L'épisode russe est le plus connu (sans doute grâce à Pouchkine et à Moussorgski) : le prince Dimitri, héritier potentiel de la couronne impériale meurt en 1598 en jouant avec des enfants de son âge, mais reparait en 1604 et revendique le trône. Assassiné après un court règne par les boïars, Dimitri réapparaît encore... Plus modestement en France en 1595, un mystérieux fils de Charles IX tente d'inquiéter Henri IV et trouve des partisans. Ce triste délire conduit François de La Ramée à la mort. Les souvenirs de la Ligue s'estompant, de telles revendications seront très rapidement confiées aux médecins et identifiées à la folie.

Au-delà de la chronique, quelles fonctions attribuer à de tels mythes ? Quel est le sens de cette même histoire répétée dans le temps et dans l'espace? D'où proviennent les constantes, le noyau commun de ces épisodes séparés ? La crédulité et l'imposture ne suffisent pas à rendre compte de la force mobilisatrice de telles aventures. Les sources littéraires, le folklore et les contes de fees permettent de saisir les ressorts inconscients de l'espérance monarchique. La bonté du roi constitue le paradigme de base ; à partir de là, toute déception peut donner lieu à une contestation de l'identité royale. Comme les héros des contes de fées, porteurs de talismans secrets mais dont le commun des mortels ignore la personnalité, le vrai roi peut être caché. Hasard d'une bataille, usurpation au berceau, volonté de pénitence, le roi peut disparaître, dans l'exil assumer les péchés de son peuple et le sauver comme le Christ a sauvé l'homme. Celui qui gouverne n'est plus qu'un usurpateur contre lequel la révolte est légitime... Toutes les espérances ne conduisent pas à l'insurrection, mais l'exaltation d'un roi prestigieux permet 
souvent et à moindre risque la critique du présent. Charlemagne, Arthur, Barberousse s'inscrivent dans la liste des « rois dormants " dont on rêve un retour salvateur.

Les fondateurs d'empire et leurs actifs propagandistes savent à leur tour utiliser les mécanismes de cette fidélité dynastique ombrageuse et fantasque. Illustrant la qualité royale prééminente, être proche du peuple et bien informé, des anecdotes répertoriées montrent Henri IV écoutant ses sujets sans se faire connaître et récompensant leur franchise bourrue. À l'inverse, le despote demeure isolé dans son palais et méprise la vox populi.

Puisant avec un égal bonheur dans des sources très différentes, Y.-M. Bercé propose un modèle d'histoire des mythologies politiques, des représentations populaires du pouvoir, centré sur l'imaginaire monarchique, mais dont l'étude de la correspondance adressée par les Français du $x^{e}$ siècle aux présidents de la République montre les avatars régaliens au-delà de la coupure républicaine.

Monique COTIRET.

Perez Zagorin, Ways of Lying : Dissimulation, Persecution, and Conformity in Early Modern Europe. Cambridge, Mass./Londres, Harvard University Press, 1990. $16 \times 24,337$ p., index.

Perez Zagorin, en choisissant pour thème de son enquête le mensonge et la dissimulation, s'est intéressé à un objet privilégié puisqu'il pose la question des conditions mêmes de possibilité de la recherche historique. En effet, comment s'assurer qu'un document dit bien ce qu'il a l'air de dire? Question corollaire : quand y a-t-il lieu d'en soupçonner la lettre pour s'interroger sur les effets qu'il vise à obtenir?

La thèse de $\mathrm{P}$. Zagorin est simple : la dissimulation, à la fois comme théorie et comme pratique, est une donnée fondamentale dans l'histoire de l'Europe moderne. L'auteur, qui propose de rebaptiser « âge de la dissimulation » (p. 330) la période qui va de 1500 au milieu du xvir siècle, montre comment la problématique du nicodémisme influence toutes les pratiques religieuses dominées: judaïsme marrane en Espagne à la fin du $x^{\mathbb{E}}$ siècle, protestantisme en Europe catholique, catholicisme en Angleterre protestante. La dissimulation présente immédiatement un double visage : les victimes de toutes les répressions l'envisagent comme moyen de défense, les dirigeants cherchent à réduire sa pratique. Sous la forme de la restriction mentale, la dissimulation est étudiée par les théologiens jésuites comme par les réformateurs amenés à se prononcer sur sa justification. L'impression générale qui se dégage de l'ensemble des textes présentés, principalement des traités de casuistique, est, à première vue, décevante : nulle loi ne peut être énoncée, sévérité et laxisme se rencontrent pareillement sous des plumes catholiques et protestantes.

Ce qui frappe, en revanche, c'est la lente substitution d'une problématique philosophique à des prémisses théologiques. Zagorin consacre son deuxième chapitre 
à passer en revue les références scripturaires et patristiques majeures invoquées dans les textes théologiques qu'il présente par la suite. Cette utile précaution n'est pas innocente : elle met en évidence la nature jurisprudentielle de la grande majorité des analyses consacrées à la restriction mentale. Pour traiter cette question, les théologiens jésuites, comme Navarrus ou Suarez, partent en quête des précédents bibliques. On voit par là que la question fait son apparition, cela dès saint Augustin, sous les espèces d'une question de droit : face à un cas de conscience, on va chercher l'avis autorisé par la tradition, quitte à céder aux séductions du probabilisme, sévèrement condamné par Pascal. Ce juridisme initial, qui sera encore perceptible chez Kant, auteur d'un opuscule Sur un prétendu droit de mentir par humanité (1797), cède la place, vers la fin du xvie siècle, à une attitude plus pragmatique. Dans l'intervalle étudié par Zagorin, on assiste à l'apparition d'un mode de questionnement proprement philosophique. La dissimulation devient un concept, par exemple chez Bacon (p. 271-274), Bodin (p. 281-288) et Hobbes (p. 327-329). L'influence de Machiavel aidant, la dissimulation est d'abord un fait et une nécessité qui donne lieu à l'analyse logique de ses conditions de possibilité et à l'analyse morale de ses effets. Au lieu de demander à une autorité si le recours à la dissimulation compromet le salut personnel, on se livre à un calcul d'intérêt rationnel.

L'accumulation de références citées constitue un épais dossier historique qui devrait fournir la mise en perspective indispensable à tout travail d'interprétation d'un texte précis. Zagorin lui-même s'y essaie, avec prudence, au fil des chapitres. C'est ainsi que la thèse défendue par Lucien Febvre à propos de la religion de Rabelais ' , thèse il est vrai déjà battue en brèche par de nombreux auteurs, semble être définitivement ruinée : Zagorin démontre, après d'autres, la possibilité de l'incrédulité comme position philosophique dès le xvie siècle (p. 289-291).

Plus globalement, cette étude permet d'esquisser la description d'une véritable " crise de la sincérité » qui submerge tous les domaines de la vie politique et intellectuelle, et qui a laissé des traces littéraires et idéologiques célèbres. On lira dans ces pages la généalogie de Dom Juan et de Iago, mais aussi celle de la figure du prêtre ou du moine dissimulateurs qui hante la propagande anticatholique du théâtre jacobéen au roman gothique et au récit sadien. Trouvant son origine historique dans la répression, la dissimulation ouvre une faille irrémédiable dans les mentalités occidentales : l'ambiguïté et l'hypocrisie coupent en deux, respectivement, les objets de l'univers naturel et les sujets du monde social. Si l'on tolère la restriction mentale, le discours oral ou écrit n'a plus d'autre critère de validité que son efficace. Tout énoncé perd sa valeur de dénotation (de mes pensées, d'un état de choses) pour devenir pur moyen au service d'une stratégie temporelle. L'apparence absorbe l'être ; la parole d'autrui, bientôt la mienne, risquent de n'être plus que le fragment trompeur d'un sens toujours incomplet. Cette perspective angoissante se dessine très tôt, puisque Zagorin en relève le pressentiment chez Mon-

1. Lucien Febvre, Le Problème de l'incroyance au $x{ }^{e} T^{e}$ siècle. La religion de Rabelais, Paris, Albin Michel, “L'évolution de l'humanité », 1968, en part. la conclusion (" Un siècle qui veut croire »), p. 419-428, mais aussi toute la deuxième partie, consacrée aux "limites de l'incroyance au $x^{e} r^{e}$ siècle ", p. 307-418. 
taigne et Bacon. Elle inquiétera tout le siècle suivant, confronté à l'inflation des serments. Que vaut un serment entaché de soupçon? Telle est la question posée, notamment en Angleterre, par bien des partisans de la tolérance.

L'enquête débouche sur des préoccupations voisines de celles exprimées par Leo Strauss dans La Persécution et l'art d'écrire ${ }^{2}$. Le fait de culture répertorié par P. Zagorin l'autorise à déceler la présence de pratiques ésotériques largement répandues. L'auteur signe ici quelques chapitres de ce qui pourrait être une histoire de la clandestinité. En particulier, l'ésotérisme des cabalistes ou des astrologues, dépouillé de ses oripeaux obscurantistes, est décrit comme un aristocratisme intellectuel qui cultive la prudence (p. 255-288). Plus généralement, l'ouvrage rejoint les thèses de Strauss en faveur de la lecture entre les lignes.

Face à la prolifération des raisons de douter, le lecteur devient-il un parfait sceptique après avoir refermé l'ouvrage ? Si c'était le cas, Zagorin, à n'en pas douter, estimerait avoir manqué son but. Cette histoire de la dissimulation fournit une typologie de ses formes qui justifie l'application du principe de raison suffisante : les documents historiques ne sont pas tous à double fond. Avant de se prononcer, par exemple, sur la duplicité de Montaigne ou de Hobbes, il faut s'assurer de deux choses : ce philosophe a-t-il quelque raison de cacher sa véritable opinion, et quels sont les moyens, historiquement attestés, dont il dispose à cette fin ? Zagorin démontre que le flair n'est pas un argument et que, en histoire des idées, une hypothèse ne perd jamais de sa séduction à être étayée par une connaissance des situations historiques concrètes.

Jean-François BaILLoN.

Jean-François Drèze, Raison d'État et raison de Dieu. Politique et mystique chez Jeanne de France. Préf. de Francis RAPP. Paris, Beauchesne, 1991. $13,5 \times 21,5,328 \mathrm{p}$.

La discrétion de Jeanne de France, furtive apparition de nos manuels, fille disgraciée de Louis XI vite répudiée par Louis XII, justifie amplement cette tentative de biographie. L'auteur, prêtre du diocèse de Digne, a travaillé en théologien cherchant les modes d'expression des systèmes de foi d'une époque, et plus encore en historien de la spiritualité, soucieux de comprendre comment une princesse devient fondatrice d'ordre et comment cet ordre, l'Annonciade, s'insère dans l'histoire monastique de son temps.

L'entreprise est bienvenue, en raison de la méconnaissance que nous avons de l'histoire des mentalités religieuses de la fin du $\mathrm{xv}^{\mathrm{e}}$ siècle, une période encore rangée dans le temps noir des abus, par des générations d'historiographes ou d'apologistes pressés. Pour compenser la mauvaise connaissance de cette période, aggravée encore par les coupures artificielles de l'histoire universitaire, l'auteur

2. Paris, Presses Pocket, "Agora ", 1989, notamment p. 55-72. 
nous introduit dans une longue, trop longue, histoire dynastique des règnes de Louis XI et Louis XII. Si les mésaventures de la princesse répudiée justifiaient une mise au point sur ce sujet, il n'en demeure pas moins qu'il n'était pas nécessaire de s'étendre aussi longuement sur une histoire politique précise mais trop classique pour apporter une compréhension nouvelle.

La seconde partie de l'ouvrage, qui s'attache à nous décrire les conditions spirituelles de la fondation de l'Annonciade, est une lecture intelligente de la spiritualité de la princesse et des problèmes religieux du temps. Il est cependant dommage que le lien entre la première et la seconde partie de la vie de Jeanne n'ait été vu qu'à travers la structure banale du renoncement au monde, que les rapports entre politique et religion n'aient pas été abordés. En dehors de la présentation du confesseur de la reine et cofondateur de l'ordre, le minime Gilbert Nicolas, tout se passe comme si l'entourage de Jeanne ne jouait aucun rôle dans la fondation. Or tout texte hagiographique, comme tout dossier de béatification, comprend des éléments sociologiques et politiques qui peuvent être décryptés par les historiens.

Bref, comme le laisse apparaître le titre, raison d'état et raison de Dieu sont juxtaposées, alors que leur articulation eût pu donner un nouvel angle d'explication de l'histoire de ce temps. Nous saurons relativement bien qui inspire la fondation, encore que la piste de Jean Gerson, celui de la Montaigne de contemplation, voire de la Théologie mystique, eût pu être mieux explorée, mais nous ne saurons pas qui paie les fondations, qui les soutient localement, qui y est recruté, ni comment et où l'Annonciade s'est diffusée. Car le succès a été indéniable, si l'on en juge par les fondations locales sans rapport direct avec le milieu berrichon comme en Rouergue.

L'auteur utilise bien la bibliographie classique (Renaudet), mais semble ignorer les travaux récents sur les réformes à la fin du $x v^{e}$ siècle et au début du $x v^{e}$ siècle, par exemple les travaux majeurs de Jean-Pierre Massaut, André Godin, Michel Veissière... qui ne sont pas seulement des études d'histoire de la théologie, mais aussi d'histoire des idées, de la spiritualité et de la société (voir, par exemple, la note 286 p. 207).

En dépit de ces critiques de fond, qui marquent la déception du chercheur, en face d'une approche historiographique un peu trop classique et étroite, ne boudons pas notre plaisir de disposer d'une étude supplémentaire sur cette période méconnue, une étude parfaitement honnête qui peut être lue agréablement et avec profit par le lecteur profane : une qualité foncière en ces temps de jargon historique pseudo-intellectuel envahissant.

Nicole Lemaitre.

Simone Goyard-Fabre, Jean Bodin et le droit de la République. Paris, Presses universitaires de France, 1989. $16 \times 24,310$ p. ("Léviathan »).

Succédant au livre de Franck Lessay, Souveraineté et légitimité chez Hobbes, ce second volume de la collection "Léviathan " que dirige S. Rials aux P.U.F. attire 
l'attention du public sur l'un des penseurs majeurs de la modernité française. Comme le soulignaient récemment A. Guéry et $\mathrm{R}$. Descimon, les six livres de $\mathbf{L a}$ République de Jean Bodin - quatorze éditions en français entre 1576 et 1629 , sans compter la version latine - peuvent être salués comme le " premier grand ouvrage de la philosophie politique française et moderne ${ }^{3}$. Entre Seyssel, Machiavel et Montesquieu, cinquante ans avant Hobbes, voici enfin la naissance de la souveraineté. En effet, précisait Lucien Jaume dans une remarquable synthèse récente, Bodin fit de la souveraineté l'« attribut par excellence de l'État moderne ", en la dotant de cette " abstraction qui allait marquer si fortement les conceptions du droit public français " ${ }^{4}$. On connaît la suite : la souveraineté, liée dans un premier temps au pouvoir royal, parvint à se reporter sur le peuple même - contrairement sans doute à toute attente. La « souveraineté populaire ", proclamée par la Révolution française, n'est-elle pas l'avatar tardif de la paradoxale continuité de l'État?

Entre la "musique des sphères " et "un droit politique que griseront les triomphes de la raison ", comme l'écrit joliment Simone Goyard-Fabre (p. 291), la place de Bodin, ainsi que son message, sont bien marqués par l'« ambiguïté ». On regrettera certes quelques mentions elliptiques : il n'est pas sûr que le « temps des rois thaumaturges " ait été aussi facilement " dépassé " (p. 12) - contrairement à ce qu'ont démontré $M$. Bloch ou J. Le Goff -, ni que le protestantisme ait représenté pour le $x^{e}{ }^{e}$ siècle une «option démocratique et libérale » (p. 31)... Mais sans doute est-ce là instruire en historien le procès d'une modernité souvent plus apparente que réelle.

Bernard COTTRET.

Henri IV et la reconstruction du royaume. Ouvr. coll., en trois sections précédées d'une préface et suivies d'une chronologie. Paris, Éd. de la Réunion des musées nationaux/Archives nationales, 1989. $21 \times 23,6,432$ p., ill.

Ce fut un plaisir de faire partie des visiteurs qui ont durant l'été dernier suivi dans l'enfilade des appartements du château de Pau le dédale du destin d'un personnage qui fut tour à tour, grâce à sa naissance et à la disparition successive de ceux qui le séparaient du pouvoir, prince de Navarre, duc de Vendôme, Henri III, roi de Navarre, et Henri IV, roi de France. Pour accompagner pareille existence et la replacer dans une époque qui porte son empreinte, il fallait un guide hors pair : il nous est offert par les maîtres d'œuvre et les auteurs de ce catalogue. L'ouvrage contient trente-neuf chapitres complétés par une chronologie henricienne, française, européenne mais non mondiale comme l'annonce le titre retenu (p. 427).

3. Alain Guéry, Robert Descimon, Histoire de la France. L'État et les pouvoirs, Paris, Seuil, 1989, p. 214.

4. Lucien J Jume, Le Discours jacobin et la démocratie, Paris, Fayard, 1989, p. 264. 
Ces trente-neuf chapitres sont autant de mises au point rédigées par des spécialistes et illustrées par des documents et une iconographie exceptionnellement rassemblés dans cette exposition, tenue à Pau puis à Paris, à l'occasion de la célébration du quatrième centenaire de l'avènement de Henri IV au trône de France.

Tous les thèmes inséparables de la fortune du bon roi sont évoqués : l'aventure de son avènement, ses retrouvailles avec Henri III, l'ultime mobilisation de la Ligue contre lui, la rédaction et le contenu de l'édit de Nantes, son entourage, ses conseillers, ses compagnons, son «image » enfin, sans oublier les circonstances de sa mort et le dèroulement de ses obsèques. $\grave{A}$ ce parcours parfaitement reconstitué par le texte et par l'image d'une vie hors du commun, viennent s'ajouter des thèmes contemporains du règne de Henri IV et plus ou moins influencés par lui : la naissance de la conscience nationale, la situation de l'armée et des armes, l'état des sciences, de la littérature, des finances, de l'économie, des voies de communication, des fortifications, de la cartographie, de l'urbanisme et de ses manifestations artistiques.

Par la nouveauté de ses approches et son souci de ne rien laisser dans l'ombre, ce catalogue est un livre d'histoire irremplaçable et magnifiquement illustré sur l'époque Henri IV.

Anne-Marie Cocula.

Pierre Castagnos, Richelieu face à la mer. Rennes, Ouest-France, 1989. $16 \times 23$, 288 p., ill., bibliogr. (" De mémoire d'homme, l'histoire ").

Le livre de P. Castagnos n'est ni une nouvelle biographie du cardinal de Richelieu, ni une synthèse sur l'histoire de la marine avant Colbert. Cet essai veut reconstituer les principes qui guident l'homme d'état dans les questions maritimes et les accommodements nécessaires de ces principes avec la réalité. L'intérêt du cardinal pour la mer s'affirme très tôt ; dès octobre 1626, en effet, il se voit conférer le titre de " Grand maitre et surintendant général de la navigation et du commerce ". Il lance trois grandes compagnies maritimes qui échouent très vite, mais serviront de modèles aux entreprises ultérieures. La difficulté du projet réside dans le décalage constant entre les ambitions du ministre, on serait tenté de dire ses rêves, et les nécessités de l'heure. La mer impose ses lois, même dans la lutte contre La Rochelle, l'Océan est un adversaire plus redoutable encore que les huguenots : le chapitre 2 dresse un tableau minutieux des échecs avant le succès final. Soulignant avec raison l'énorme part de contraintes qui s'imposent à Richelieu, P. Castagnos nous entraine loin des flots bleus, et décrit les aspects de la terrible guerre de Trente ans, les révoltes populaires, les conspirations des grands... Le projet initial semble abandonné et c'est grand dommage.

Monique Corrret. 
Lucien Bely, Espions et ambassadeurs au temps de Louis XIV. Paris, Fayard, 1990. $15,3 \times 23,5,905 \mathrm{p}$.

Le vaste travail de L. Bély constitue un plaidoyer pour une histoire diplomatique, une histoire internationale des volontés politiques qui n'ignore pas le destin des peuples. Les négociations qui entourent la fin de la très longue et cruelle guerre de Succession d'Espagne et aboutissent à la paix d'Utrecht se révèlent un terrain particulièrement fécond pour saisir «le passage d'une guerre à outrance vers une paix équilibrée » mais aussi « le saut autrement plus ambitieux de l'exaltation du héros conquérant vers celle de l'homme cosmopolite " (p. 13). L'enquête qui couvre l'ensemble de l'Europe, s'articule en trois temps : 1. la quête de l'information y compris par l'espionnage ; 2 . le mode de vie des négociateurs et l'art de la diplomatie; 3. le pacifisme qui marque l'aube des Lumières. De l'anecdotique à l'idéologique, tous les textes sont traqués et interrogés comme autant de signes d'un cosmopolitisme à venir.

Le monde de l'espionnage est décrit par touches successives et portraits entrecroisés. Aventuriers de haute volée comme les Chavigny (p. 123-125), belles dames peu farouches, faux moines et véritables indicateurs se côtoient sous le regard soupçonneux de la police. Ce monde de l'ombre demeure bien difficile à cerner; les sources renvoient davantage aux hantises des polices qu'aux motivations des espions. Si «l'argent, la femme et la foi » (chapitre 5) se rencontrent à l'origine de bien des vocations, les espions ne livrent pas aisément leur âme. Dans un monde de fidélités enchâssées, où commence la trahison? On peut regretter l'absence de distinction nette entre " renseigner " et " espionner " proprement dit, mais on aurait tort de bouder son plaisir, entre jus de citron et encre sympathique, ces fragments de vies qui surgissent du passé ont tout le piquant et la saveur nostalgique des romans inachevés.

Les négociateurs offrent à l'historien une emprise plus solide : 450 négociateurs ont été recensés de 1697 à 1715 , depuis l'ambassadeur extraordinaire jusqu'au simple intermédiaire, du grand seigneur au secrétaire... Pour l'ensemble des pays européens, le poids de la noblesse est prépondérant, l'Angleterre et les ProvincesUnies introduisent tout juste « un début de diversité ». On rencontre de véritables lignées familiales de négociateurs. Les structures diplomatiques se perfectionnent, le droit international progresse et l'on voit apparaitre une véritable " civilisation de la diplomatie ». Pourtant la diversité l'emporte encore dans la formation des négociateurs, pluralité des cultures, mais aussi des comportements sociaux, officiers, ecclésiastiques, courtisans ne réagissent pas de la même manière. La France innove avec la création d'une Académie politique (ancêtre de l'E.N.A.) qui de 1712 à 1719 est censée former les futurs diplomates. À leur programme : étude de la correspondance diplomatique, initiation à l'art de la conférence à partir des dépêches, formation d'un style écrit, latin obligatoire, mais aussi italien, espagnol et anglais... Dubois supprime sous la Régence cette institution protégée par Torcy.

Isolés dans un pays étranger, les ambassadeurs témoignent des différences entre les nations, c'est le sens du fameux « comment peut-on être persan? ». Mais rassemblés pour travailler, les diplomates engendrent une nouvelle sociabilité, inventent des formes nouvelles qui «mettent en valeur les points communs et 
gomment les différences" (p. 410). Le minutieux règlement des conférences est organisé pour éviter les interminables querelles de préséance : c'est le "pêlemêle " qui fait table rase des rangs des différents états européens, qui les nivelle dans l'attente de la reconstruction à venir. Gestes policés, paroles mesurées, temps suspendu, les dignitaires sont en représentation ; dans ce monde de l'apparence, ils parlent français alors même que s'impose la suprématie anglaise...

Par sa longueur, l'importance des différents fronts, les intérêts mondiaux engagés, la guerre de Succession d'Espagne marque l'apogée de la guerre au xvII $^{\circ}$ siècle, mais cet excès même suscite la contestation (peut-être pourrait-on risquer un parallèle avec 14-18 ?). Les hommes d'Utrecht sont portés par les attentes et les espoirs de l'opinion. Le Projet de paix perpétuelle de l'abbé de Saint-Pierre est publié l'année de la conférence. Les nations sont à la recherche d'un nouvel ordre international. Après la " paix romaine " et la fugace " paix de Dieu ", une idéologie de la paix est invoquée. Les questions religieuses ne sont plus au premier rang des préoccupations, contrairement aux assemblées de Westphalie. Selon Lucien Bély, les nouvelles valeurs morales et politiques - paix, bonheur, nature, liberté - seraient nées de ce "vide" : "les congrès donnaient une dimension internationale à la culture des lumières comme une réponse aux questions posées par la " crise" de la conscience européenne. Les certitudes politiques et sociales remplaçaient l'inquiétude religieuse, et peut-être métaphysique. Le cosmopolitisme trouvait sa raison et sa fin : une organisation rationnelle, raisonnée et raisonnable de la chrétienté » (p. 745). Transformer les négociateurs d'Utrecht en " missionnaires » de cette " foi nouvelle » et en disciples de l'abbé de Saint-Pierre me paraît bien optimiste... Par ailleurs, l'idéologie de la paix, en France, est largement propagée par Fénelon et ses amis les dévots. Mais Lucien Bély a parfaitement saisi un moment d'histoire. Le livre se termine par un vibrant appel en faveur d'une « irénologie historique [...] au moment où l'Europe des nations efface ses frontières à l'ouest et espère le dégel à l'est ». Appel entendu, semble-t-il...

Monique CotrRet.

Gioacchino Gargallo di Castel Lentini, Storia della storiografia moderna. I. Il Settecento. Terza edizione. Rome, Bulzoni, 1990. $17 \times 24,347$ p., index.

Premier volume d'une vaste enquête sur l'historiographie moderne, cet ouvrage porte sur l'ensemble du xviII siècle. C'est un travail extrêmement pédagogique, qui cherche à donner au lecteur des points de repère clairs et précis, l'abondance des références permettant d'articuler à des auteurs très célèbres des textes qui ont été davantage oubliés. Au fil des chapitres, l'Auteur étudie cinq conceptions de l'historiographie, correspondant approximativement à un déroulement chrono- 
logique; il analyse à chaque fois le type de méthode historique utilisée, en recherchant ses présupposés philosophiques ou politiques. Le premier chapitre, intitulé " la constitution de l'apparat philosophique et la nouvelle historiographie politique », examine l'apparition d'une nouvelle historiographie dans les premières années du $\mathrm{xvIII}^{\mathrm{e}}$ siècle. L'historiographie se sépare de l'apologie religieuse, telle qu'elle a pu être pratiquée par Bossuet, elle se libère des contraintes imposées par la Contre-Réforme : on commence à chercher à construire l'histoire en utilisant des présupposés qui ne soient pas chrétiens, en étudiant, par exemple, la vertu des païens. Sur un plan méthodologique, se posent les questions du pyrrhonisme historique et du type de certitude que l'on peut obtenir en histoire, questions auxquelles Bayle ou Saint-Evremond cherchent à apporter des réponses. L'historiographie libertine accumule des travaux érudits, en s'inspirant des recherches effectuées à la Renaissance. Le chapitre II, intitulé " histoire des institutions et recherche des origines ", étudie un nouveau courant historiographique, de type juridico-érudit, animé par un sentiment national. Ce courant a été représenté en France par Saint-Simon dont les recherches historiques ont eu pour but de justifier les privilèges de la noblesse, et surtout par Boulainvilliers qui chercha à fonder historiquement sa thèse de l'antique liberté des Francs. Avec le même type de méthode, Dubos utilisa toute son érudition pour lutter contre Boulainvilliers en dénonçant l'illégitimité des droits seigneuriaux et en critiquant les usurpations de la noblesse aux $\mathrm{xx}^{\mathrm{e}}$ et $\mathrm{x}^{\mathrm{e}}$ siècles pour justifier la suprématie de la monarchie absolue de César à Louis XIV. Cette historiographie juridico-érudite a été également représentée en Italie par Giannone et Vico, et en Allemagne par Gottfried Arnold. Le chapitre III, " les lumières et leur histoire ", est consacré à l'œuvre de Montesquieu, de Voltaire et de Hume. Si Montesquieu est un admirateur de Boulainvilliers, il ne pratique pas du tout la même méthode historique. Ses observations essaient de mettre en évidence des liens de causalité, de rechercher une critique interne des situations, grâce à une expérience politique. Les œuvres historiques de Voltaire, tout comme celles de Hume, permettent d'analyser comment des mobiles politiques se sont introduits dans des discours proprement historiques. L'Auteur voit ainsi dans les thèses de Voltaire sur le progrès une polémique contre le péché originel, ou dans son portrait de Louis XIV une apologie du despote éclairé, capable de réformer l'État suivant un plan rationnel. Le chapitre $\mathrm{IV}$, intitulé " historiographie de la culture et de l'aliénation », travaille sur un courant allemand représenté entre autres par Mosheim, Möser, Herder. L'Auteur étudie en particulier les thèses de Mosheim sur les antiques mours des Saxons, sur leurs vertus sauvages laissées inaltérées par les Romains et sur la théorie des libertés germaniques. Enfin, le dernier chapitre, intitulé " histoire et sociologie ", s'intéresse essentiellement aux thèses de Turgot et de Condorcet sur les progrès de l'humanité, thèses qui ont influencé toute la conception de l'historiographie sous la Restauration. La clarté des analyses, la précision des références, l'abondance des notes, la présence d'un index font de ce livre un instrument de travail extrêmement utile. 
Joël Félxx, Les Magistrats du Parlement de Paris (1771-1790). Dictionnaire biographique et généalogique. Paris, Sedopols, 1990. $17 \times 24,240$ p.

L'expérience judiciaire du chancelier Maupeou constitue un des temps forts de la vie politique du $x^{\prime}{ }^{e}{ }^{e}$ siècle : vaste tentative de despotisme éclairé remise en cause par l'ampleur de la contestation. J. Félix livre ici une étude sur les hommes qui ont accepté de peupler les nouvelles cours. Cette analyse est d'autant plus intéressante que ces magistrats ont été traînés dans la boue par leurs contemporains : en cette époque de contestation de l'arbitraire royal, il ne faisait pas bon être du côté du roi! Les partisans de Maupeou ont été jugés incompétents, d'une probité douteuse et raillés pour leur modeste origine sociale. J. Félix démontre avec force l'inanité de tels propos : «Les magistrats du parlement Maupeou avaient donc beaucoup plus de similitudes que de différences avec les officiers exilés. Pour la plupart ils étaient bien nés. Aisés ou riches, sans être tous des millionnaires, ils appartenaient pleinement à ce monde de la robe dont les niveaux de fortune étaient extrêmement variables mais assez faibles relativement aux négociants ou aux fermiers généraux de la capitale. Ils étaient enfin cultivés et parfois même érudits » (p. 42). Dès lors pourquoi avoir choisi de soutenir l'expérience du chancelier? Faut-il y voir, comme le prétendent nombre de pamphlétaires, une revanche du parti des dévots, de la cour et des jésuites? Plus fondamentalement, J. Félix révèle que les hommes du chancelier sont les hommes du roi : «... les magistrats du parlement Maupeou étaient sans doute plus directement liés au pouvoir royal qu'au monde judiciaire. En effet, pour nombre d'entre eux, leurs grands-parents avaient fait partie de la maison du roi ou des maisons des princes et des princesses de la famille royale. " Le formidable affrontement qui entoure le " coup d'état " Maupeou "s'inscrit dans la lutte entre la monarchie administrative et la monarchie judiciaire ". Les magistrats du parlement Maupeou, loin d'être des ambitieux parvenus, ont eu le courage de braver l'opinion et de servir une monarchie pas toujours reconnaissante.

Avant le dictionnaire proprement dit, une seconde étude plus rapide est consacrée au devenir des parlementaires après l'épisode révolutionnaire. Le divorce entre les patriotes et les parlementaires remonte à l'arrêté du 25 septembre 1788 qui prévoit la réunion des États Généraux selon les formes usitées en 1614... Le 14 octobre 1790, les membres de la chambre des vacations de Paris se réunissent pour rédiger une protestation virulente, et secrète, contre la destruction des parlements... À la fin de 1793, cette déclaration est dénoncée et les anciens magistrats prennent le chemin de l'échafaud : parmi les 130 officiers pourvus sous le règne de Louis XVI et encore en vie en 1789 « 13 d'entre eux furent guillotinés, une quarantaine décrétés d'arrestation, une vingtaine emprisonnés, 42 émigrés ou portés émigrés... ". Mais, en dépit de ces drames, les continuités semblent bien l'emporter, comme en témoigne le destin de la famille Sabatier (p. 75).

$\mathrm{Au}$ total un travail précieux pour qui veut comprendre la fin de l'Ancien Régime et le premier $\mathrm{XIX}^{\mathrm{e}}$ siècle. 
Carol Buum, Rousseau and the Republic of Virtue. The Language of Politics in the French Revolution. Ithaca/Londres, Cornell University Press, 1986. $15,8 \times 23,5,302$ p., bibliogr., index.

L'Auteur s'attaque dans cet ouvrage au vieux et difficile problème des origines intellectuelles de la Révolution et du rôle qu'ont pu jouer les Philosophes dans sa préparation. Pour Carol Blum, il y a continuité entre le système politique de Rousseau et la Terreur. C'est la thèse de Taine revue par Cochin (jamais cité) et Talmon (The Origins of Totalitarian Democracy, New York, 1960), mais éclairée d'un jour nouveau car, anticipant sur ce qui s'est écrit depuis, l'Auteur se refuse à tout jugement d'ordre moral et s'en tient à l'analyse des concepts. C'est celui de vertu qui fait ici les frais de la démonstration : Rousseau, dès Genève, s'est pensé comme un modèle de vertu et c'est cette même vertu qu'il enseigne à travers ses livres; cependant, entre la vertu rêvée et l'immoralité constatée, le décalage est grand. Ce constat résume son drame : si l'homme est bon (et Rousseau est cet homme-là), les hommes, malheureusement, sont méchants. D'où la solitude grandissante de Jean-Jacques et la tentation de la violence (p. 202, «Ô vertu, le poignard, seul espoir de la terre / est ton arme sacrée "). Comme on le verra avec Robespierre et Saint-Just, vertu et violence sont indissociablement liées. Si la thèse n'est pas nouvelle, comme je l'ai rappelé, elle s'accompagne dans cet ouvrage de nombreuses analyses souvent pertinentes (ainsi le rapport de Catherine Théot et Robespierre, p. 265 et suiv.) et d'intéressants chapitres sur la réception de l'image de Rousseau pendant la période révolutionnaire. Il est clair que, pour Carol Blum, Rousseau n'est pas responsable de la Terreur, mais son cuvre l'explique. On ne saurait, dans le cadre d'une recension, engager une discussion - d'autant que la littérature sur le sujet est considérable; mais je crois qu'en se focalisant sur la notion de vertu l'auteur se condamnait à un flou conceptuel invalidant ce qu'il fallait démontrer : d'abord parce que la vertu ne caractérise pas plus l'œuvre de Rousseau que celle des lecteurs de Plutarque qui sont pléthore dans le siècle; ensuite, parce que Rousseau se pensait bon - ce qui n'est pas la même chose - et parfois seulement vertueux. Au total, l'ouvrage que déparent quelques approximations (p. 120, Mlle Lambercier est la cousine de Rousseau ; " Bérère ", p. 143, etc.) est cependant une contribution intéressante à l'histoire des représentations du rousseauisme : malheureusement, il n'aide ni à comprendre Rousseau (lu de loin), ni à expliquer la Révolution (vue de près) - et encore moins la Terreur. Car, comme écrivait Rousseau dans un texte que l'Auteur ne parait pas connaître : «Quand on veut étudier les hommes, il faut regarder près de soi ; mais pour étudier l'homme il faut apprendre à porter sa vue au loin " (Essai sur l'origine des langues); ce que Bolivar avait compris à sa manière lorsqu'il disait: "Pour comprendre les révolutions et ceux qui y participent, il faut à la fois les observer de très près et les juger de très loin. " La vertu n'explique pas plus Rousseau, que le rousseauisme la Terreur. 
Guy Chaussinand-Nogaret, La Vie quotidienne des femmes du roi. D'Agnès Sorel à Marie-Antoinette. Paris, Hachette, 1990. $13 \times 20,265$ p. (« La vie quotidienne »).

Si le corps du roi ne cesse d'interroger les historiens, l'étude des reines demeure trop souvent fragmentée, légère et anecdotique. Le royaume de France ne devant pas tomber en quenouille, les épouses royales n'accèdent habituellement à la curiosité historienne que lorsqu'elles deviennent régentes et investissent la noble sphère du politique. L'ouvrage de Guy Chaussinand-Nogaret rompt heureusement avec une telle tradition et s'intéresse aux " femmes du roi... pluriel agressif, qui peut paraître bien singulier ", selon la jolie formule de l'auteur. Au-delà des références frivoles ou grivoises, il s'agit d'une réflexion sur la société de cour, d'une analyse dans le temps de la répartition des rôles et des fonctions entre l'épouse légitime et la (ou les) favorite(s). À la reine revient la stabilité, l'ordre et la continuité dynastique. Autour de la dame de beauté, s'ordonnent les fêtes et les plaisirs ; c'est le mouvement, l'effervescence créatrice, la célébration artistique et littéraire, le « ministère de l'idéal "...

Henri IV semble un instant vouloir confondre les deux registres en épousant sa maîtresse. La mort de Gabrielle, dont les obsèques reprennent un ancien cérémonial réservé aux reines (une effigie de cire revêtue d'une robe pourpre et d'un voile de satin blanc offerte pendant trois jours à la contemplation des grands...) met fin au scandale et évite un imbroglio juridique bien dangereux : qui des aînés légitimés ou des cadets nés après le mariage eût été le dauphin?

Louis XIV expérimente une complexe polygamie : trois femmes officielles lui semblent nécessaires, la reine et deux favorites, La Vallière et Montespan, Montespan et Fontanges, Maintenon et Montespan... Dans cette curieuse architecture, la maîtresse en titre, surchargée d'honneurs et de dignités, n'est bien souvent déjà plus la dame de coeur. Guy Chaussinand-Nogaret donne de cette trilogie une intéressante interprétation : elle «symbolisait l'incarnation, par le truchement de la femme, d'une triple légitimité, légitimité du corps mystique du roi, par la reine, instrument de l'éternité monarchique ; légitimité de l'image allégorique du souverain, par la favorite officielle témoin du dédoublement de la personne royale et de sa double nature christique, homme et dieu à la fois ; légitimité du sentiment et du sexe, par la dame du moment, de l'être trivial et périssable, contingent et borné, auquel est reconnu le droit d'échapper au symbole et de revêtir par instant son humanité. Admirable construction qui justifie jusqu'en ses égarements l'emblématique royale " (p. 157).

Bien peu chrétienne, l'attitude du roi ne choque que les dévots et les clercs scrupuleux, l'Église soupire après sa conversion, mais d'un tel héros peut-on réellement attendre qu'il se plie à la morale ordinaire? Il faudra le xvII ${ }^{e}$ siècle et la désacralisation de la monarchie pour que les amours de Louis XV deviennent péchés scandaleux, et que l'on soupçonne le crime derrière les grilles d'un mythique harem royal. L'inébranlable fidélité de Louis XVI est encore plus mal appréciée, c'est l'absence de favorite qui concentre sur Marie-Antoinette les haines et les injures. Loin de partager la célèbre condamnation de Chateaubriand qui voyait dans le règne des favorites « une des calamités de l'ancienne monar- 
chie ", Guy Chaussinand-Nogaret constate l'étroite "concordance » entre le dépérissement de l'institution des favorites et celui du prestige royal : « la disparition de l'écran protecteur permit de frapper la monarchie au cour » (p. 26).

Monique CotrRet.

Pour une histoire politique. Sous la dir. de René Rémond. Paris, Seuil, 1988. $14 \times 20,5,403$ p., index ("L'univers historique »).

Ce livre, paru sous la direction de René Rémond, rassemble nombre de chercheurs de la génération active, de Jean-Pierre Azéma à Pierre Milza, Antoine Prost, Jean-Pierre Rioux, Jean-François Sirinelli, Michel Winock et illustre ce phénomène historique contemporain assez remarquable : la réintégration, après un long discrédit, des faits politiques dans le champ d'observation de l'histoire.

Que l'histoire politique renaisse témoigne à la fois d'un regain d'intérêt, d'une évolution des recherches et des réflexions des historiens sur leur discipline. C'est sans doute cette dernière qui fut déterminante, tant il est vrai qu'elle ne rend compte que de fluctuations proprement intellectuelles: l'interrogation sur le métier d'historien, la volonté $d^{\prime}$ '《atteindre les réalités véritables» (René Rémond).

C'est de cette réflexion menée dans le cadre de la revue Annales par Marc Bloch et Lucien Febvre qu'est sortie ce que l'on a appelé la " nouvelle histoire ", en réaction à une histoire jugée par eux superficielle, à laquelle l'histoire politique fut identifiée. Il est vrai qu'elle se limitait trop souvent à l'analyse des crises ministérielles ou de la personne des souverains; liée à l'histoire de l'État, elle subit le discrédit de celui-ci et l'interdit jeté dans l'historiographie et les sciences sociales pendant trente ans sur son étude, au profit des réalités économiques, sociales et culturelles.

Le renouvellement de l'histoire politique porte aujourd'hui sur la conception même du politique et de son histoire; l'initiative vient d'historiens formés au contact d'autres disciplines comme la sociologie et la science politique; il explique à la fois l'enrichissement du concept et l'élargissement du champ politique. La politique, ce sont à la fois des idées, des langages, des comportements, qui entretiennent des relations avec d'autres aspects de la réalité, l'économie, la religion ou la guerre. L'historien ne peut donc se réduire à l'« analyste du passé » qui permettrait au politologue de dégager des lois; sa démarche implique des comparaisons à travers l'espace et la durée qui permet de comprendre des comportements politiques qui sont le fruit d'une culture enracinée dans notre longue histoire. S'interroger sur les parts respectives de la continuité et de la nouveauté par une mise en perspective, tel est l'apport essentiel de l'histoire à la compréhension de la politique.

Non que tout soit politique; les frontières de la politique ne sont pas établies définitivement; certaines questions sont au ccur de la vie politique quand d'autres sont en retrait. Ainsi la « question religieuse ", les rapports entre l'Église 
et l'État occupaient-ils une place centrale au $\mathrm{XIx}^{\mathrm{e}}$ siècle, bien plus importante qu'aujourd'hui. La régulation de ce marché aux limites imprécises est effectuée à la fois par la communauté nationale dans sa totalité et par le pouvoir dont les décisions s'imposent à celle-ci.

Le livre ne se réduit pas cependant à un plaidoyer pour une histoire politique ayant atteint sa phase de maturité. Il constitue aussi un inventaire de l'état de la discipline, de ses méthodes, de ses connaissances, de ses résultats. L'histoire politique a su s'adapter aux méthodes d'autres sciences sociales, comme le montre la variété des sujets abordés : l'association, la guerre, les partis, les élections, la biographie...; elle est bien «... le point où confluent la plupart des activités et récapitule les autres composantes de l'ensemble social ».

Parmi les enjeux cernés et les problématiques renouvelées, en dépit des contours fluctuants du politique - plus insaisissable à mesure que son poids devient plus réel - on retiendra un juste hommage au père de la géographie électorale, André Siegfried. Trente ans après la mort de ce grand havrais, il n'est pas inutile de rappeler son œuvre, à la fois géographique et politique, comme en témoigne son magistral Tableau politique de la France de l'Ouest paru en 1913, qui montrait la diversité des « tempéraments " politiques et l'ancienneté de leur enracinement; ainsi que le note René Rémond, d'« André Siegfried à François Goguel et à Alain Lancelot la ligne est continue qui signale une veine dans laquelle le monde entier voit une spécialité de l'école française d'histoire politique $"$.

C'est cette école, essentiellement représentée dans le livre par les universitaires de l'Institut d'études politiques de Paris et de l'université de Paris X, qui établit une nouvelle cartographie du politique, loin des définitions réductrices et des affirmations dogmatiques; si l'identité du politique demeure difficile à cerner, si les sujets traités constituent en fait des repères autonomes dont les interférences sont quelquefois mal élucidées, si des questions demeurent en suspens, les recherches menées à l'étranger ou en France aboutissent à cette synthèse utile, riche à la fois des acquis de la discipline et des hypothèses qui orientent son avenir.

L'ouvrage arrive, enfin, à point nommé. Ainsi que le démontre justement René Rémond, " aucune affirmation n'est aussi contraire à l'évolution que celle d'une prétendue dépolitisation [...]», au contraire, il relève " ... l'élévation progressive du niveau de compréhension ainsi que les exigences du public en matière d'information politique ». Intérêt et exigence : «... parce qu'il récapitule les autres niveaux de la réalité, le politique est une des expressions les plus hautes de l'identité collective [...] ce qu'on appelle parfois la culture politique et qui résume la singularité du comportement d'un peuple n'est pas un élément entre autres du paysage politique; c'est un puissant révélateur de l'ethos d'une nation et du génie d'un peuple. " Que ce recueil d'historiens nous invite aussi brillamment à lire la politique à ce niveau est un bon signe dans le présent de la République.

Tristan LeCOQ. 
Hannah Arendt, La Nature du totalitarisme. Trad. de l'anglais et préf. par Michelle-Irène B. de Launay. Paris, Payot, 1990. 14 × 22,5, 183 p. (« Bibliothèque philosophique Payot »).

Sous le titre La Nature du totalitarisme, l'ouvrage de $\mathrm{H}$. Arendt qui nous est offert présente trois articles. Deux relatifs au statut de la compréhension du phénomène totalitaire et un troisième renvoyant au rapport entre religion et politique. Véritables mises au point, ces articles permettent de lever deux ambiguïtés. La première concernant la compréhension du phénomène totalitaire prisonnier de l'alternative, qui a tendance à en faire un accident quand elle n'en fait pas un effet nécessaire de l'histoire. La seconde concernant la définition du phénomène totalitaire comme phénomène religieux. $\mathrm{H}$. Arendt le précise avec netteté. Le fait de se demander si le phénomène totalitaire est un accident ou un effet nécessaire est pervers, car cette façon de vouloir trouver ainsi une explication totale de l'histoire ne conduit qu'à nous faire manquer l'événement. Aussi nous propose-t-elle de renverser la démarche et de retourner à l'événement lui-même, en procédant comme Montesquieu, par l'analyse des structures et des mobiles qui meuvent les systèmes politiques. En l'occurrence, s'agissant du totalitarisme, celui-ci a été un régime inédit fondé sur la crainte et aboutissant à "l'esseulement " de l'humanité.

La même chose vaut quant au rapport religion-politique. Se demander si le totalitarisme a été ou non une religion est une autre façon d'en masquer la nature et de ne pas voir où se situe sa véritable portée religieuse, à savoir la perte de la crainte de l'enfer.

En fait, à travers deux angles différents, $H$. Arendt fait preuve de la même vigueur méthodologique et du même souci de poser les bonnes questions, nous donnant ainsi les moyens de voir en quoi quelque chose comme la destruction totalitaire du sens commun fait événement et situe le totalitarisme bien au-delà d'un simple accident ou d'un effet logique de l'histoire.

Bertrand Vergely.

\section{CULTURE POLITIQUE ET ENSEIGNEMENT}

Anthony Grafion, Forgers and Critics. Creativity and Duplicity in Western Scholarship. Princeton, NJ, Princeton University Press, 1990. 14,5 × 22,3, 157 p., index.

Ce court volume développe une "Public Lecture " donnée par l'Auteur à l'université de Princeton. Sur un problème précis (celui de la relation entre le faussaire 